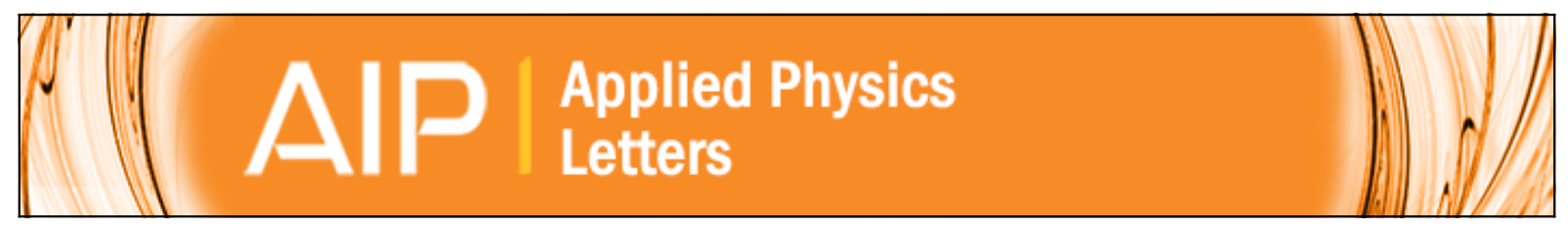

Electric preconcentration and detection of latex beads with interdigitated electrodes

Roberto de la Rica, César Fernández-Sánchez, and Antonio Baldi

Citation: Applied Physics Letters 90, 174104 (2007); doi: 10.1063/1.2731311

View online: http://dx.doi.org/10.1063/1.2731311

View Table of Contents: http://scitation.aip.org/content/aip/journal/apl/90/17?ver=pdfcov

Published by the AIP Publishing

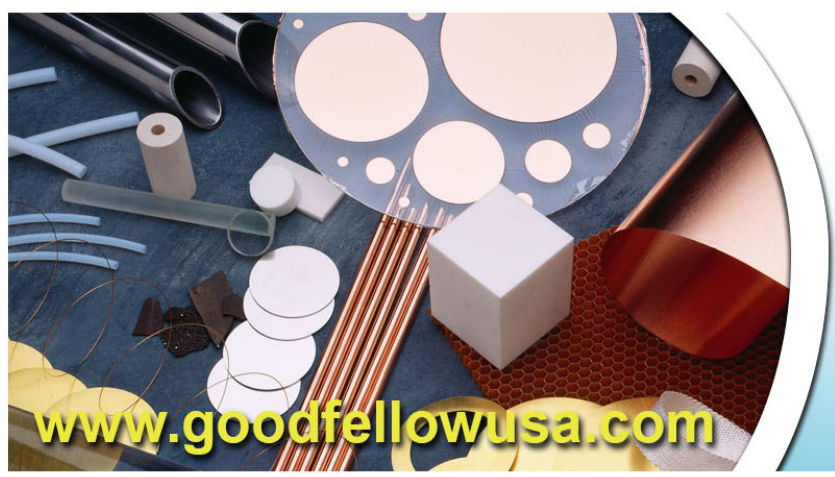

BODIFEllOU metals • ceramics • polymers composites $\cdot$ compounds $\cdot$ glasses

Save $5 \% \cdot$ Buy online 70,000 products $\cdot$ Fast shipping 


\title{
Electric preconcentration and detection of latex beads with interdigitated electrodes
}

\author{
Roberto de la Rica, ${ }^{\text {a) }}$ César Fernández-Sánchez, and Antonio Baldi \\ Centro Nacional de Microelectrónica (IMB-CNM, CSIC), Esfera UAB, Campus UAB, \\ 08193 Cerdanyola del Vallés, Barcelona, Spain
}

(Received 7 March 2007; accepted 24 March 2007; published online 23 April 2007)

\begin{abstract}
Latex beads are adhered to the surface of a chip containing polycrystalline silicon interdigitated electrodes by positive dielectrophoresis. The presence of the microparticles induces a change in the distribution of the electric fields and currents that can be detected by measuring either the conductance or the capacitance at the electrode terminals. This combination of actuation and detection with a single device is proposed as a suitable system applicable to biosensing.
\end{abstract}

(C) 2007 American Institute of Physics. [DOI: 10.1063/1.2731311]

Bead-based sensors are becoming common tools in bioanalytical chemistry. One of the most popular approaches uses paramagnetic particles as mobile substrates, where captured biomolecules are immobilized. A magnetic field is applied in order to attract the beads to the transducer surface. As a result of a biorecognition event, a signal, either electrochemical, ${ }^{1}$ optical, ${ }^{2}$ or magnetoresistive, ${ }^{3,4}$ is generated. Magnetoresistive detection systems are gaining considerable interest in recent years. ${ }^{5}$ The recognition between the capture element and the analyte results in the attachment of the beads to the sensor surface, where the presence of the magnetic particles is detected by a change in the resistivity of the material. Giant magnetoresistive devices, ${ }^{6}$ anisotropic magnetic resistive rings, ${ }^{7}$ Hall effect sensors, ${ }^{8-10}$ as well as spin valves ${ }^{11}$ and magnetic tunnel junction structures ${ }^{12,13}$ have been proposed as suitable transducers for this kind of measurements.

In the present work, an alternative approach based on electric preconcentration and detection of latex beads is presented and tested. It consists in the accumulation and adsorption of the microparticles at the surface of a pair of interdigitated electrodes by positive dielectrophoresis, followed by their impedimetric detection. The electrodes are made of polycrystalline silicon. Their capability of measuring both the conductivity $(\sigma)$ and the permittivity $(\varepsilon)$ of the solution where they are immersed has been previously demonstrated. ${ }^{14}$ The conductance $(G)$ and the capacitance $(C)$ measured at the electrodes are inversely proportional to these two magnitudes, respectively, by the cell constant $(k)$ :

$$
\begin{gathered}
C=\frac{\varepsilon}{k}, \\
G=\frac{\sigma}{k} .
\end{gathered}
$$

The cell constant depends on both the geometry of the electrodes and the geometry of the space between the electrodes filled with the solution. The presence of insulating objects at the electrode surface alters the electric field distribution and the path of currents, thus changing the geometry of the cell (Fig. 1). This variation can be monitored by a change in the measured conductance or capacitance. The magnitude of the change in the cell constant depends on both the size of the insulating objects and their surface coverage. Translated to the system under study, beads with larger radius give rise to a larger signal as individuals, leading to a more sensitive detection, whereas smaller particles lead to a higher amount of beads per unit area, thus allowing a wider dynamic range.

Dielectrophoresis refers to the force on polarizable particles in a spatially nonuniform electric field and provides a suitable method for manipulating microparticles in liquid suspension. Two types of forces can be exerted: particles can be pulled towards points of maximum electric field (termed positive dielectrophoresis) or pushed towards locations of minimum electric field (termed negative dielectrophoresis). Here, positive dielectrophoresis is used in order to accumulate beads at the electrode fingers, where the electric field lines converge and the presence of the insulating microparticles has a greater impact on the cell constant (Fig. 2). Latex beads remain adhered to the surface after the dielectrophoretic force is turned off. ${ }^{15}$ This dielectrophoretically driven adhesion of beads emulates microparticle attachment by a biorecognition event, like in magnetoresistive biosensors. ${ }^{5}$ Interdigitated electrodes with $3 \mu \mathrm{m}$ width fingers separated by $10 \mu \mathrm{m}$ and $3 \mu \mathrm{m}$ diameter latex beads are used in this work. Electrodes with similar electric field penetration depth have been previously used in dielectrophoretic accumulation of latex particles. ${ }^{15,16}$

In order to enhance the adhesion of beads, the sensors were grafted with 3-aminopropyltrymethoxysilane in a gasphase procedure, by closing them in a previously nitrogen saturated chamber containing the silane, overnight. Hydrocarbonated chains in the silane render the surface more hydrophobic, promoting van der Waals interactions with the particles, and the terminal amino groups provide positive charges that can interact with negative counterparts present at the bead surface. Dielectrophoresis was performed after applying a drop of $0.25 \%(w / v)$ beads in $250 \mathrm{mM}$ glycine buffer, which has a conductivity of $15 \mu \mathrm{S} / \mathrm{cm}$. Amino-acidbased buffers have been previously used as low conductivity media in on-chip electrophoresis experiments ${ }^{17,18}$ and protein detection biosensors. ${ }^{19}$ A peak-to-peak potential of $1 \mathrm{~V}$ was applied, and the frequency was set at $5 \mathrm{kHz}$. Figure 3 shows images of the electrodes after different accumulation times. 


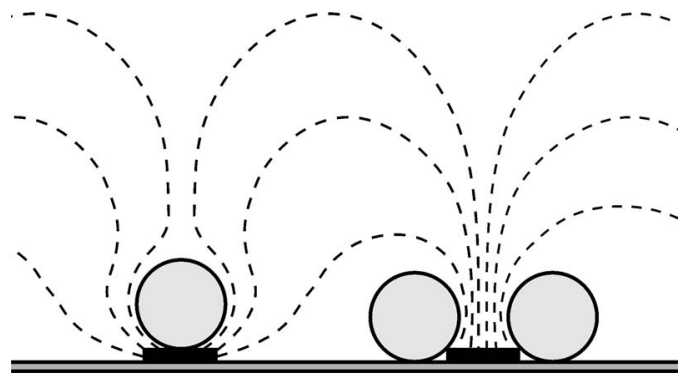

FIG. 1. Schematic representation of the detection system. Particles are attracted to the points of maximum electric field, changing the field lines distribution.

As expected, beads preferably adhere to the surface of the electrodes, either at the edges or on top. All the particles present in a certain area were counted in order to assess the on-chip bead density. These pictures clearly demonstrate that the proposed electrodes are able to concentrate micron-sized latex particles by positive dielectrophoresis.

Variation in the electrical properties of the cell caused by the presence of the microparticles was evaluated with the following experiment. An impedance spectrum of the beadmodified electrodes was taken from $1 \mathrm{MHz}$ to $10 \mathrm{kHz}$ applying a $10 \mathrm{mV}$ amplitude excitation signal, in $250 \mathrm{mM}$ glycine buffer, after each accumulation step. The spectra were fitted to an equivalent circuit in order to obtain the resistance and the capacitance of the solution. Details about the fitting procedure have been published elsewhere. ${ }^{14}$ Resistance values were converted to conductance ones by simply inverting them. Results were translated into percentages, taking the plain sensor without beads as a reference. A parallel blank experiment was also performed by repeating the dielectrophoresis with another sensor in a solution containing no beads. These measurements monitor changes in the magnitudes of interest due to other factors, such as temperature drift or carbon dioxide diffusion, and were subtracted from the former ones. Figure 4 shows a plot of the variation in conductance and capacitance versus bead density. Saturation at high bead density values is caused by accumulation in the space between the fingers, where the impact of the insulating particles in the geometry of the cell is lower than that at the electrode surface. Both capacitance and conductance measurements give rise to comparable plots, as expected from a change in the cell constant, with a $2 \times 10^{3}$ bead $^{-1} \mathrm{~mm}^{2}$ slope

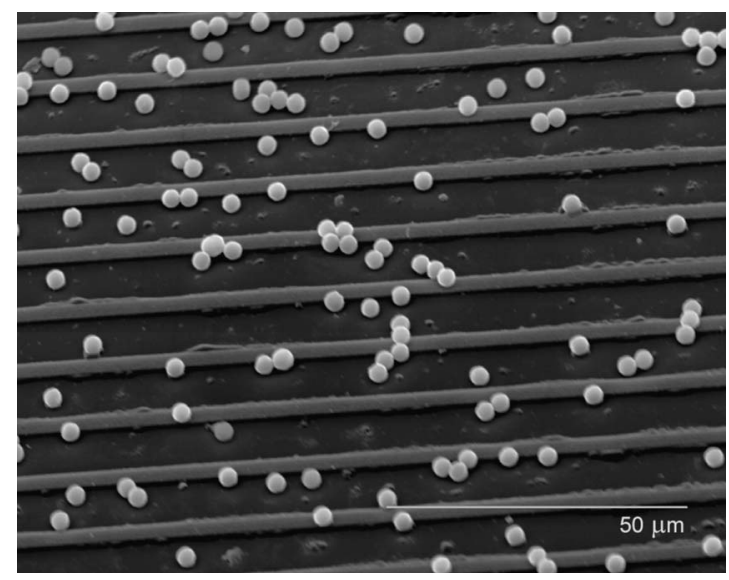

FIG. 2. Scanning electron microscopy image of the $3 \mu \mathrm{m}$ width beads adhered at the sensor surface.

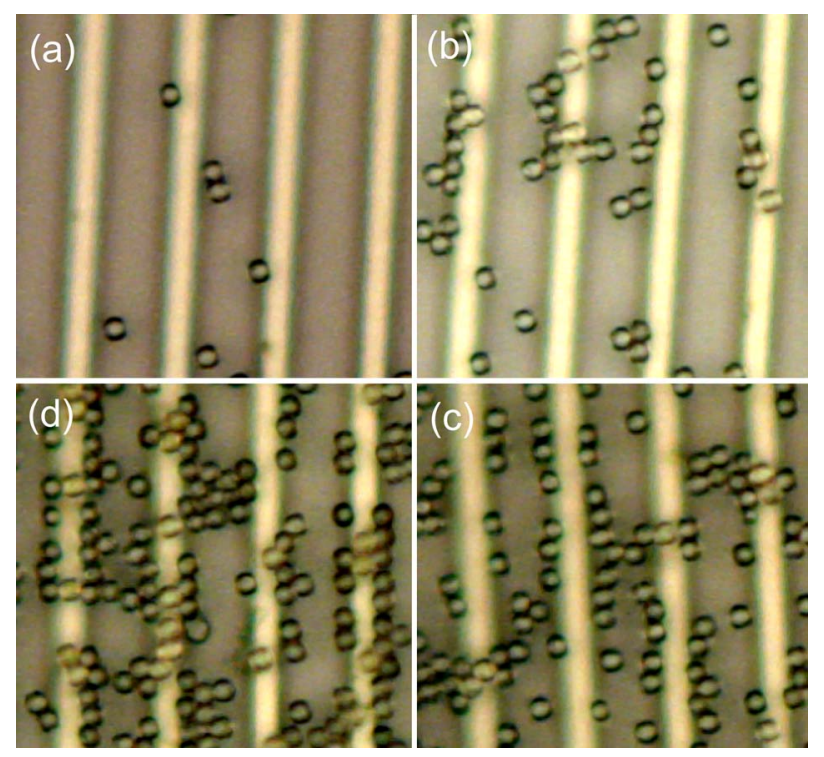

FIG. 3. Images of bead-coated electrodes with (a) 0.2, (b) 2, (c) 3, and (d) $8 \times 10^{3} \mathrm{~mm}^{-2}$ bead density.

in the linear part of the curve. These experiments prove that $3 \mu \mathrm{m}$ diameter insulating microparticles can be detected using interdigitated electrodes with the proposed geometry.

In conclusion, a bead-based electric actuation and transduction system has been presented and tested. This approach is comparable to magnetoresistive biodetection, that is, dielectrophoretic accumulation substitutes magnetic preconcentration and impedimetric transduction replaces magnetoresistive detection. Polycrystalline silicon interdigitated electrodes can be fabricated with complementary metaloxide-semiconductor technologies and modified with biomolecules, i.e., antibodies. ${ }^{19}$ Latex beads modified with a wide variety of capture biomolecules are commercially available. Thereby, a biosensor may be easily developed, the analyte acting as a bridge between the transducer surface and the dielectrophoretically preconcentrated beads. Combination of these electrodes with microfluidics for bead manipulation

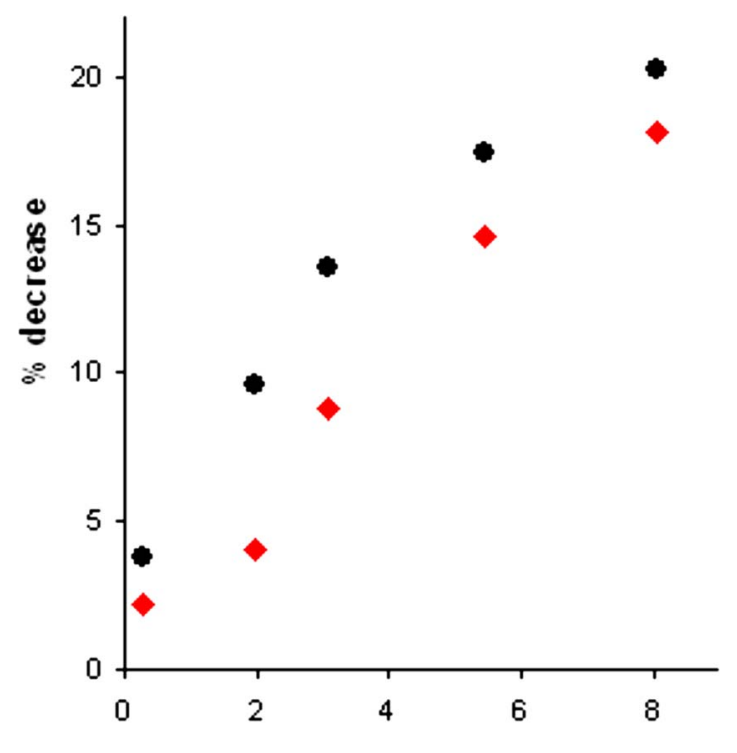

$\left(\times 10^{-3}\right)$ beads $\mathrm{mm}^{-2}$ 
makes the proposed detection system an appealing alternative for fully integrated biosensors and laboratory-on-chip systems.

Two of the authors (C.F.-F. and A.B.) acknowledge the Spanish Ministry of Science and Education for the award of a Ramón y Cajal contract.

${ }^{1}$ V. N. Goral, N. V. Zaytseva, and A. J. Baeumner, Lab Chip 6, 414 (2006). ${ }^{2}$ S. Dubus, J. F. Gravel, B. Le Drogoff, P. Nobert, T. Veres, and D. Boudreau, Anal. Chem. 78, 4457 (2006).

${ }^{3}$ M. Brzeska, M. Panhorst, P. B. Kamp, J. Schotter, G. Reiss, A. Pühler, A. Becker, and H. Brückl, J. Biotechnol. 112, 25 (2004).

${ }^{4}$ G. Kurlyandskaya and V. Levit, Biosens. Bioelectron. 20, 1611 (2005).

${ }^{5}$ D. L. Graham, H. A. Ferreira, and P. P. Freitas, Trends Biotechnol. 22, 455 (2004).

${ }^{6}$ R. L. Edelstein, C. R. Tamanaha, P. E. Sheehan, M. M. Miller, D. R. Baselt, L. J. Whitman, and R. J. Colton, Biosens. Bioelectron. 14, 805 (2000).

${ }^{7}$ M. M. Miller, G. A. Prinz, S.-F. Cheng, and S. Bounnak, Appl. Phys. Lett. 81, 2211 (2002).

${ }^{8}$ P. A. Besse, G. Boero, M. Demierre, V. Pott, and R. Popovic, Appl. Phys. Lett. 80, 4199 (2002).
${ }^{9}$ L. Ejsing, M. F. Hansen, and A. K. Menon, Appl. Phys. Lett. 84, 4729 (2004).

${ }^{10}$ G. Mihajlović, P. Xiong, and S. von Molnár, Appl. Phys. Lett. 87, 112502 (2005).

${ }^{11}$ L. Lagae, R. Wirix-Speetjens, J. Das, D. Graham, H. Ferreira, P. P. Freitas, G. Borghs, and J. De Boeck, J. Appl. Phys. 91, 7445 (2002).

${ }^{12}$ W. F. Shen, X. Y. Liu, D. Mazumdar, and G. Xiao, Appl. Phys. Lett. 86, 253901 (2005).

${ }^{13}$ S. G. Grancharov, H. Zeng, S. H. Sun, S. X. Wang, S. O'Brien, C. B. Murray, J. R. Kirtley, and G. A. Held, J. Phys. Chem. B 109, 13030 (2005).

${ }^{14}$ R. de la Rica, C. Fernández-Sánchez, and A. Baldi, Electrochem. Commun. 8, 1239 (2006).

${ }^{15}$ J. Auerswald, D. Widmer, N. F. De Rooij, A. Sigrist, T. Staubli, T. Stöckli, and H. F. Knapp, Electrophoresis 26, 3697 (2005).

${ }^{16}$ N. Gadish and J. Voldman, Anal. Chem. 78, 7870 (2006).

${ }^{17}$ C. F. Edman, D. E. Raymond, D. J. Wu, E. Tu, R. G. Sosnowski, W. F. Butler, M. Nerenberg, and M. J. Heller, Nucleic Acids Res. 25, 4907 (1997).

${ }^{18}$ R. G. Sosnowski, E. Tu, W. F. Butler, J. P. O'Connell, and M. J. Heller, Proc. Natl. Acad. Sci. U.S.A. 94, 1119 (1997).

${ }^{19}$ R. de la Rica, A. Baldi, and C. Fernández-Sánchez, Appl. Phys. Lett. 90, 074102 (2007). 